\title{
Post-COVID-19 Pulmonary Fibrosis: Report of Two Cases
}

\author{
Authors: \\ Saurabh Karmakar, *Priya Sharma, Ameet Harishkumar, Deependra \\ Kumar Rai \\ Department of Pulmonary Medicine, All India Institute of Medical Sciences, Patna, \\ India \\ *Correspondence to priyasharma25292@gmail.com \\ Disclosure: $\quad$ The authors have declared no conflicts of interest. \\ Received: $\quad 09.12 .20$ \\ Accepted: $\quad 01.04 .21$ \\ Keywords: $\quad$ Acute hypoxic respiratory failure, COVID-19, pulmonary fibrosis. \\ Citation: $\quad$ EMJ Respir. 2021; DOI/10.33590/emjrespir/20-00270
}

\begin{abstract}
COVID-19 caused by severe acute respiratory syndrome coronavirus 2 has led to a pandemic situation worldwide. However, remarkable progress has been made in evolving policies, strengthening healthcare efforts, and pharmacotherapy. As more patients are recovering from COVID-19, clearer concepts about possible short- and long-term complications are emerging. Respiratory failure is the most common morbidity in hospitalised patients, and post-COVID-19 pulmonary fibrosis is the most common respiratory complication after recovery. The authors report two cases of COVID-19 pneumonia with respiratory failure who were cured but developed pulmonary fibrosis with restrictive lung disease in the follow-up period.
\end{abstract}

\section{INTRODUCTION}

COVID-19 is defined as an illness caused by the novel coronavirus severe acute respiratory syndrome coronavirus 2 (SARS-CoV-2). COVID-19 pneumonia has unique features and comprises co-occurrence of viral pneumonia and acute respiratory distress syndrome." The authors describe the cases of two patients with COVID-19 pneumonia who were hospitalised with respiratory failure and subsequently developed pulmonary fibrosis. To the best of the authors' knowledge, this case report is the index publication from their country describing two cases of post-COVID-19 pulmonary fibrosis, in which a restrictive pattern was demonstrated on spirometry. The authors seek to draw the

attention of clinicians towards understanding the natural course of COVID-19 in patients and to identify and treat sequelae.

\section{CASE REPORT}

\section{Case One}

A non-smoking, 52-year-old male presented with cough on and off and myalgia associated with fever and dyspnoea for 5 days. Nasopharyngeal and throat swab sampling for real-time reverse transcription (RT)-PCR assay for the detection of SARS-CoV-2 was advised by a local practitioner, which was positive, and was referred to the authors' institution. On admission, the patient had a high fever. Clinical findings and vitals at the time of presentation are provided in Table 1. 
Table 1: Clinical and laboratory parameters of both cases.

\begin{tabular}{|l|l|l|}
\hline & Case One & Case Two \\
\hline $\begin{array}{l}\text { Oxygen saturation in room air at time } \\
\text { of presentation }\end{array}$ & $91 \%$ & $93 \%$ \\
\hline Auscultatory findings & $\begin{array}{l}\text { Fine crepitation in bilateral infra- } \\
\text { scapular and infra-axillary lung fields }\end{array}$ & $\begin{array}{l}\text { Fine crepitation in right scapular, } \\
\text { bilateral infra-scapular, and infra- } \\
\text { axillary lung fields }\end{array}$ \\
\hline Inflammatory markers & 65 & 52 \\
\hline ESR (O-30 mm/hr) & 195 & 131 \\
\hline CRP $(0-5 \mathrm{mg} / \mathrm{L})$ & 1,366 & 721 \\
\hline Ferritin $(22-322 \mathrm{ng} / \mathrm{mL})$ & 1,903 & 1,408 \\
\hline LDH $(230-460 \mathrm{U} / \mathrm{L})$ & $>20.00$ & 2.79 \\
\hline D-dimer $(<0.2 \mathrm{mg} / \mathrm{L})$ & 0.15 & 0.05 \\
\hline Procalcitonin $(<0.50 \mathrm{ng} / \mathrm{mL})$ & &
\end{tabular}

CRP: C-reactive protein; ESR: erythrocyte sedimentation rate; LDH: lactate dehydrogenase.

All routine blood investigations and inflammatory markers were performed and repeated as per clinical requirements (Table 1). Bedside chest $\mathrm{X}$-ray revealed peripheral haziness.

The patient was started on moist $\mathrm{O}_{2}$ along with intravenous injection of $6 \mathrm{mg}$ dexamethasone, twice daily subcutaneous injection of 60 $\mathrm{mg}$ enoxaparin, and injection of $200 \mathrm{mg}$ remdesivir on the initial day, followed by $100 \mathrm{mg}$ administered intravenously for 5 days. On Day 12, his oxygen saturation $\left(\mathrm{SpO}_{2}\right)$ dropped to $85 \%$ on $15 \mathrm{~L} / \mathrm{min}$ supplied via a non-rebreathing mask. Consequently, he was started on non-invasive ventilation using a mechanical ventilator with $100 \%$ fraction of inspired oxygen. The patient was also treated with intravenous injection of 1 $\mathrm{mg} / \mathrm{kg}$ body weight methylprednisolone in two divided doses. Intravenous injection of $8 \mathrm{mg} / \mathrm{kg}$ body weight tocilizumab was administered on Day 14, as per institutional protocol. He was on non-invasive ventilation intermittently until Day 21 , followed by a gradual decrease in fraction of inspired oxygen, and was later changed to non-rebreathing mask support. CT pulmonary angiography with high-resolution CT (HRCT) thorax on Day 25 revealed multiple confluent areas of consolidation and ground-glass opacities (GGO) with posterobasal predominance and peribronchial distribution. Traction bronchiectasis with no evidence of pulmonary embolism was also visible (Figure 1A). His $\mathrm{O}_{2}$ requirement gradually reduced. Repeat nasopharyngeal and throat swab testing by RT-PCR assay for SARSCoV-2 was performed after 3 weeks and was negative. A screening HRCT thorax after 6 weeks of admission showed multiple patchy areas of perilobular and subpleural consolidation and GGOs with interstitial thickening and fibrotic changes, including traction bronchiectasis and subpleural bands (Figure 1B). Spirometry using the EasyOne ${ }^{\circledR}$ spirometer (NDD Medizintechnik AG, Zurich, Switzerland) was performed after 6 weeks of onset of illness, revealing moderate restriction. He was discharged on long-term oxygen therapy, a course of oral steroids (prednisolone $10 \mathrm{mg}$ once daily) and antifibrotics (pirfenidone $600 \mathrm{mg}$ three times daily), and called for review at a future date. 

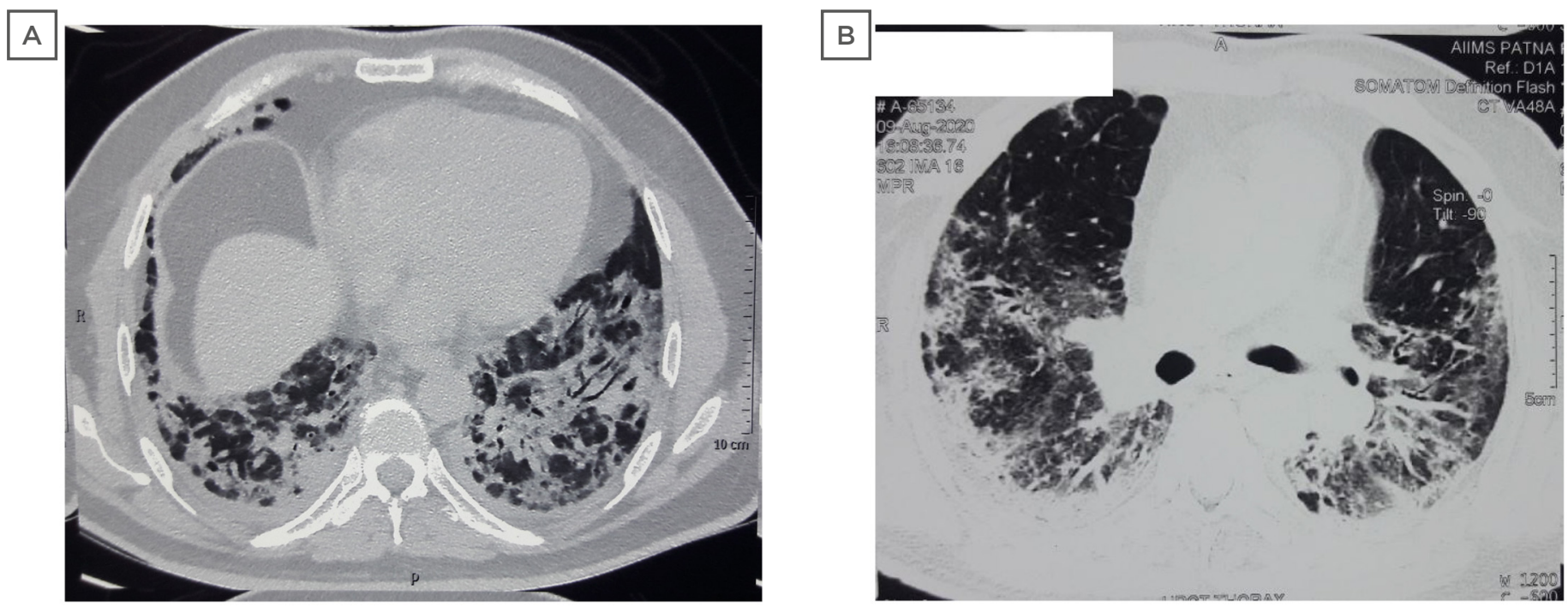

Figure 1: Pre- and post-COVID-19 high-resolution CT images: Case One.
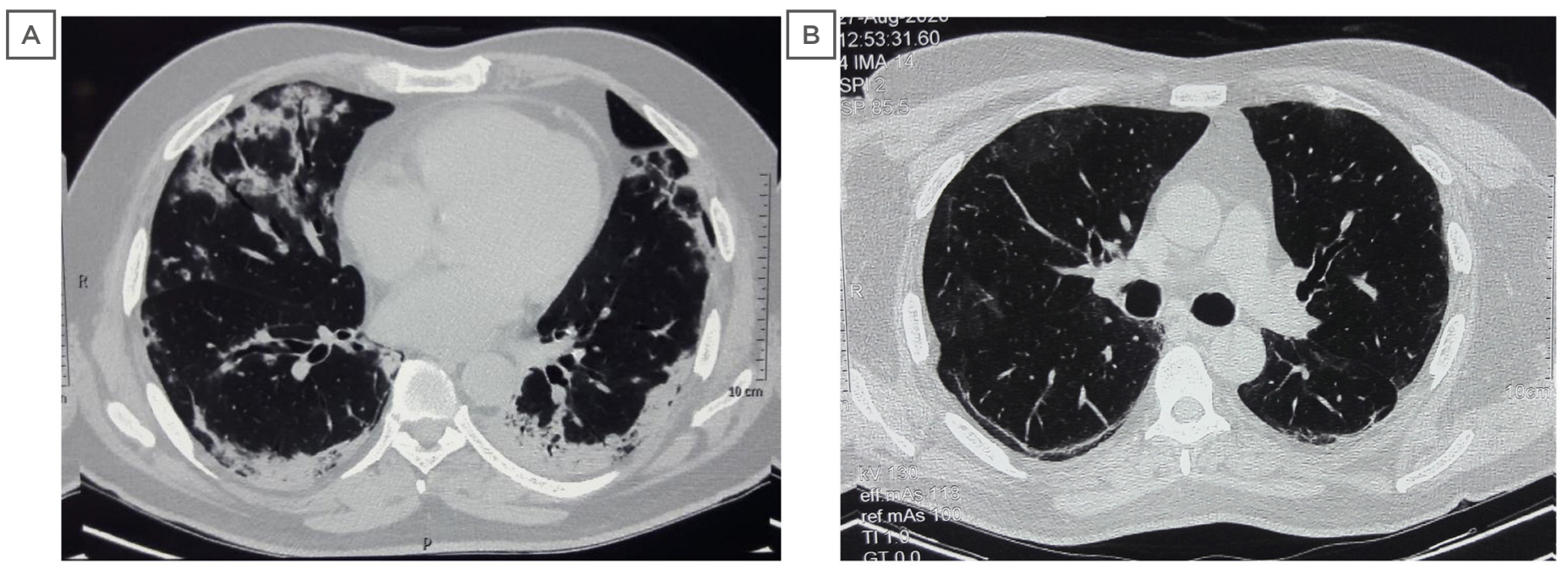

Figure 2: Pre- and post-COVID-19 high-resolution CT images: Case Two.

\section{Case Two}

A 57-year-old male with no history of smoking and a history of hypertension on telmisartan presented with cough on and off for 7 days and fever for 1 day. A RT-PCR assay of SARS-CoV-2 was performed, which was positive, and was referred to the authors' institution. On admission, clinical examination and vitals were recorded as shown in Table 1.

The patient was started on dexamethasone and enoxaparin injections. After 3 days of admission, his room-air $\mathrm{SpO}_{2}$ fell to $89 \%$ and moist $\mathrm{O}_{2}$ was given at $5 \mathrm{~L} / \mathrm{min}$ by venturi mask. Injection of remdesivir was administrated for 5 days. After 7 days of admission, his room-air $\mathrm{SpO}_{2}$ was $84 \%$ and $\mathrm{O}_{2}$ at $12 \mathrm{~L} / \mathrm{min}$ was delivered using a nonrebreathing mask. Injection of dexamethasone was stopped after 10 days. After 14 days of admission, his room-air $\mathrm{SpO}_{2}$ improved to $90 \%$, followed by $94 \%$ after 21 days. CT pulmonary angiography with HRCT thorax was performed on Day 21, which showed areas of consolidation and GGOs predominantly in the posterobasal lobes with subpleural sparing, areas of bronchiectasis and bronchiolectasis, and minimal areas of early honeycombing in the basal areas. There was no 
evidence of pulmonary embolism (Figure 2A). Repeat nasopharyngeal and throat swab testing by RT-PCR assay for SARS-CoV-2 was negative.

The patient was discharged on Day 28 of admission, with a room-air $\mathrm{SpO}_{2}$ of $96 \%$ and on oral steroids (prednisolone $10 \mathrm{mg}$ once daily) and antifibrotics (pirfenidone $600 \mathrm{mg}$ tablets three times daily). He was reviewed in the outpatient department, 3 weeks after discharge, and a repeat HRCT thorax (Figure $2 \mathrm{~B}$ ) and spirometry was done, which showed mild restriction. His treatment was continued and he was called for review at a future date.

\section{DISCUSSION}

Coronaviruses are a group of related RNA viruses that cause diseases in mammals and birds. In humans, they cause respiratory tract infections that can range from mild to lethal. Lethal varieties can cause SARS-CoV, Middle East respiratory syndrome coronavirus (MERS-CoV), and COVID-19.

Presentations of COVID-19 are categorised as asymptomatic, mild-to-moderate (81.0\%), severe (14.0\%), critically ill (5.0\%), and fatal (overall 2.3\%). ${ }^{2}$

Fibrotic abnormalities of the lung have been detected after 3 weeks of onset of symptoms in mild, moderate, and severe disease. ${ }^{3}$ Restriction, reduced diffusion capacity, and small airways obstruction have been identified at discharge and 2 weeks later. $^{3}$ The impairments are mainly restrictive in nature, with a predominance of abnormalities in diffusing capacity of the lungs for carbon monoxide, vital capacity, and total lung capacity, compared to forced expiratory volume in 1 second. ${ }^{5}$ Lung function abnormalities are more common among elderly patients and patients with severe COVID-19 and high levels of inflammatory markers. These individuals show evidence of pulmonary fibrosis, such as interstitial thickening, coarse reticular patterns, and parenchymal bands. ${ }^{4}$ A meta-analysis by Ahmed et al. ${ }^{5}$ showed that in SARS-CoV and MERS-CoV (coronavirus outbreaks similar to the current COVID-19 pandemic), although lung function improves over time, reduction in diffusing capacity of the lungs for carbon monoxide may still be present in $11-45 \%$ of survivors at 12 months.
Under normal circumstances, the wound healing response is downregulated after injury is repaired. Infection with SARS-CoV in mouse models and humans causes severe damage to epithelial cells lining the airways. When the infectious burden overwhelms the system, the wound healing response can become dysregulated, resulting in scarring and fibrosis. ${ }^{6}$ Epidermal growth factor receptor (EGFR) signalling is one of the key regulators of the wound healing process. Studies of SARS-CoV in mouse models have shown that EGFR ligands are expressed at the onset of wound healing (Day 2 post-infection) and return to baseline after the resolution of injury (Day 9 post-infection), coinciding with the expression of wound healing genes at Day 2 and downregulation of these genes by Day 9. In mouse models with constitutively active EGFR, SARS-CoV infection causes enhanced lung disease and the EGFR ligands amphiregulin and heparin-binding EGFlike growth factor are upregulated. Exogenous addition of these ligands during infection leads to enhanced lung disease and altered wound healing dynamics. Dysregulation in the initial activation or the eventual downregulation of this pathway disrupts the wound healing process, resulting in unchecked fibrosis. ${ }^{7}$ The combined effect of the virus-induced cell injury and inflammatory mediators may be responsible for the lung damage that occurs in COVID-19.8

Predictors of pulmonary fibrosis are advanced age, illness severity, length of intensive care unit (ICU) stay and mechanical ventilation, smoking, and chronic alcohol use. ${ }^{9}$ Older people are more likely to have severe symptoms and be more susceptible to both SARS-CoV-2 and its complication of lung fibrosis. ${ }^{10}$ Experimental studies using mouse models have reported increased lung fibrosis in older animals compared to younger ones." Additionally, there is an increased resistance of fibroblasts and myofibroblasts to apoptosis in older mouse models. ${ }^{12}$ Illness severity is correlated with inflammatory markers, including lactate dehydrogenase (LDH) levels following acute lung injuries, and is also an indicator of pulmonary tissue destruction..$^{13}$ Concomitantly, the extent of lung injury and inflammatory response correlates with the extent of fibroblastic response..$^{14,15} \mathrm{~A}$ high LDH level was found to significantly correlate with the risk of pulmonary fibrosis following MERS-CoV infection. ${ }^{16}$ One follow-up study at 
6 months in patients with SARS-CoV showed a significant relationship between elevated levels of LDH during acute illness and an increased risk of developing pulmonary fibrosis. ${ }^{13}$ Higher disease severity is closely related to the prolonged length of ICU stay and mechanical ventilation, and leads to possible ventilator-induced lung injury. In ventilator-induced lung injury, there occurs a release of pro-inflammatory modulators, worsening acute lung injury, and increased mortality or pulmonary fibrosis in survivors. ${ }^{17}$ Smoking is associated with chronic oxidative stress, increased expression of inflammatory cytokines, and interstitial lung fibrosis, with the risk continuing even after cessation. ${ }^{18,19}$ Smokers are more likely to have severe symptoms, need ICU admission and mechanical ventilation, or die compared to non-smokers. ${ }^{20}$ Chronic alcohol use is a predisposing factor for severe respiratory infections and acute respiratory distress syndrome..$^{21,22}$ Chronic alcohol use causes glutathione depletion, chronic oxidative stress, inflammation, and induction of TGF- $\beta$ in the lungs, thereby increasing the risk of acute lung injury and pulmonary fibrosis. ${ }^{22}$ TGF- $\beta$ is a potent fibroproliferative cytokine.

Tocilizumab appears to be an effective treatment option in patients with COVID-19 and a risk of cytokine storms. ${ }^{23}$ Pirfenidone could inhibit apoptosis, downregulate expression of angiotensin-converting enzyme 2 receptors, decrease inflammation by several mechanisms, and ameliorate oxidative stress, thus protecting pneumocytes and other cells from COVID-19 invasion and cytokine storm simultaneously. ${ }^{24}$

Both patients with COVID-19 pneumonia had high inflammatory markers and progressed to respiratory failure, with Case One needing noninvasive ventilation, and Case Two needing supplemental $\mathrm{O}_{2}$ with pharmacotherapy. Repeat RT-PCR became negative, signifying viral clearance and resolution of COVID-19. Signs of resolution in CT thorax begin approximately 2 weeks after the onset of initial symptoms. ${ }^{25}$ Pulmonary fibrosis develops in most patients with SARS-CoV-2 after 5 weeks. ${ }^{26}$ In the present cases, screening with HRCT thorax showed radiological signs of fibrosis and spirometry as well as a finding of restriction after 6 weeks, leading to the hypothesis of post-COVID-19 pulmonary fibrosis. Optimal time for follow-up imaging to assess for radiological clearance is, as yet, unknown. ${ }^{27}$

Post-COVID-19 pulmonary fibrosis is a predictable and serious complication of COVID-19 pneumonia that requires early recognition and comprehensive management. Future research should attempt to address the issues of prevalence and significance of post-COVID-19 pulmonary fibrosis.

\section{LIMITATIONS}

Clinical investigations were performed in a public sector institution and therefore, under government regulations during the pandemic period, investigations beyond spirometry and CT chest were prohibited for patients. The 6-minute walk distance test could not be performed; however, it is part of a future original research article in patients with post-COVID-19 fibrosis. Although follow-up was until 6 weeks, fibrosis was evident by the radiographic findings on repeat CT scan and restrictive findings on spirometry, after repeat RT-PCR testing was negative for SARSCoV-2, suggesting viral clearance. Although a longer follow-up would be advantageous, the authors believe that the short-term follow-up findings would be a beneficial addition to the relevant literature on post-COVID-19 fibrosis.

\section{References}

1. Gibson PG et al. COVID-19 acute respiratory distress syndrome (ARDS): clinical fea-tures and differences from typical pre-COVID-19 ARDS. Med J Aust. 2020;213(2):54-6.

2. Wu Z, McGoogan JM. Characteristics of and important lessons from the coronavirus disease 2019 (COVID-19) outbreak in China: summary of a report of 72314 cases from the Chinese Center for Disease Control and Prevention. JAMA. 2020;323(13):1239-42.

3. Raghu G, Wilson KC. COVID-19 interstitial pneumonia: monitoring the clinical course in survivors. Lancet Respir Med. 2020;8(9):839-42.

4. Yu M et al. Prediction of the development of pulmonary fibrosis using serial thin-section
CT and clinical features in patients discharged after treatment for COVID-19 pneumonia. Korean J Radiol. 2020;21(6):746-55.

5. Ahmed $\mathrm{H}$ et al. Long-term clinical outcomes in survivors of severe acute respiratory syndrome and Middle East respiratory syndrome coronavirus outbreaks after hospi-talization or ICU admission: a systematic review 
and meta-analysis. J Rehabil Med. 2020;52(5):jrm00063.

6. Wynn TA. Common and unique mechanisms regulate fibrosis in various fibroprolif-erative diseases. J Clin Invest. 2007;117:524-9.

7. Venkataraman T et al. Overactive epidermal growth factor receptor signaling leads to increased fibrosis after severe acute respiratory syndrome coronavirus infection. J Virol. 2017;91:e00182-17.

8. Liu $\mathrm{J}$ et al. Overlapping and discrete aspects of the pathology and pathogenesis of the emerging human pathogenic coronaviruses SARS-CoV, MERS-CoV, and 2019-nCoV. J Med Virol. 2020;92:491-4.

9. $\mathrm{Xu} \mathrm{J}$ et al. Use of senescenceaccelerated mouse model in bleomycin-induced lung injury suggests that bone marrow-derived cells can alter the outcome of lung injury in aged mice. J Gerontol A Biol Sci Med Sci. 2009;64(7):731-9.

10. Huang WT et al. Plasminogen activator inhibitor 1 , fibroblast apoptosis resistance, and agingrelated susceptibility to lung fibrosis. Exp Gerontol. 2015;61:62-75.

11. Ojo AS et al. Pulmonary fibrosis in COVID-19 survivors: predictive factors and risk reduction strategies. Pulm Med. 2020;2020:6175964.
12. Nikolich-Zugich J et al. SARSCoV-2 and COVID-19 in older adults: what we may expect regarding pathogenesis, immune responses, and outcomes. Geroscience. 2020;42(2):505-14.

13. Wong KT et al. Severe acute respiratory syndrome: thinsection computed tomog-raphy features, temporal changes, and clinicoradiologic correlation during the con-valescent period. J Comput Assist Tomogr. 2004;28(6):790-5.

14. Strieter RM, Mehrad B. New mechanisms of pulmonary fibrosis. Chest. 2009;136(5):1364-70.

15. Wallace WAH et al. Inflammationassociated remodelling and fibrosis in the lung: a process and an end point. Int J Exp Pathol. 2007;88(2):103-10.

16. Das KM et al. Follow-up chest radiographic findings in patients with MERS-CoV after recovery. Indian J Radiol Imaging. 2017:27(3):342-9.

17. Oeckler RA, Hubmayr RD. Ventilatorassociated lung injury: a search for better therapeutic targets. Eur Respir J. 2007;30:1216-26.

18. Strzelak A et al. Tobacco smoke induces and alters immune responses in the lung triggering inflammation, allergy, asthma and other lung diseases: a mechanistic re-view. Int J Environ Res Public Health 2018;15(5):1033.
19. Spira A et al. Effects of cigarette smoke on the human airway epithelia cell tran-scriptome. Proc Natl Acad Sci USA. 2004;101(27):10143-8.

20. Vardavas Cl, Nikitara K. COVID-19 and smoking: a systematic review of the evi-dence. Tob Induc Dis 2020;18:20.

21. Kershaw CD, Guidot DM. Alcoholic lung disease. Alcohol Res Health. 2008;31:66-75

22. Sueblinvong $\vee$ et al. Chronic alcohol ingestion primes the lung for bleomycin-induced fibrosis in mice. Alcohol Clin Exp Res. 2014;38(2):33643.

23. Luo $\mathrm{P}$ et al. Tocilizumab treatment in COVID-19: a single center experience. J Med Virol. 2020;92(7):814-8.

24. Seifirad S. Pirfenidone: a novel hypothetical treatment for COVID-19. Med Hypothe-ses. 2020;144:110005.

25. Pan $\mathrm{F}$ et al. Time course of lung changes at chest CT during recovery from corona-virus disease 2019 (COVID-19). Radiology. 2020;295(3):715-21.

26. Antonio GE et al. Thin-section CT in patients with severe acute respiratory syn-drome following hospital discharge: preliminary experience. Radiology. 2003;228(3):810-5.

27. George PM et al. Respiratory follow-up of patients with COVID-19 pneumonia. Thorax. 2020;75(11). 